\title{
Mean-cluster approach indicates cell sorting time scales are determined by collective dynamics
}

\author{
Carine P. Beatrici, ${ }^{1,2}$ Rita M. C. de Almeida, ${ }^{1,3}$ and Leonardo G. Brunnet ${ }^{1, *}$ \\ ${ }^{1}$ Instituto de Física, Universidade Federal do Rio Grande do Sul, Av. Bento Gonçalves 9500, \\ C.P. 15051, 91501-970 Porto Alegre, RS, Brazil \\ ${ }_{2}^{2}$ Programa de Computação Científica, Fundação Oswaldo Cruz, Av. Brasil, 4365, Manguinhos, Rio de Janeiro, C.P. 21040-360, Brazil \\ ${ }^{3}$ Instituto Nacional de Ciência e Tecnologia, Sistemas Complexos Rua Dr. Xavier Sigaud, 150, Urca, Rio de Janeiro, C.P. 22290-180, Brazil
}

(Received 10 December 2015; revised manuscript received 26 October 2016; published 2 March 2017)

\begin{abstract}
Cell migration is essential to cell segregation, playing a central role in tissue formation, wound healing, and tumor evolution. Considering random mixtures of two cell types, it is still not clear which cell characteristics define clustering time scales. The mass of diffusing clusters merging with one another is expected to grow as $t^{d / d+2}$ when the diffusion constant scales with the inverse of the cluster mass. Cell segregation experiments deviate from that behavior. Explanations for that could arise from specific microscopic mechanisms or from collective effects, typical of active matter. Here we consider a power law connecting diffusion constant and cluster mass to propose an analytic approach to model cell segregation where we explicitly take into account finite-size corrections. The results are compared with active matter model simulations and experiments available in the literature. To investigate the role played by different mechanisms we considered different hypotheses describing cell-cell interaction: differential adhesion hypothesis and different velocities hypothesis. We find that the simulations yield normal diffusion for long time intervals. Analytic and simulation results show that (i) cluster evolution clearly tends to a scaling regime, disrupted only at finite-size limits; (ii) cluster diffusion is greatly enhanced by cell collective behavior, such that for high enough tendency to follow the neighbors, cluster diffusion may become independent of cluster size; (iii) the scaling exponent for cluster growth depends only on the mass-diffusion relation, not on the detailed local segregation mechanism. These results apply for active matter systems in general and, in particular, the mechanisms found underlying the increase in cell sorting speed certainly have deep implications in biological evolution as a selection mechanism.
\end{abstract}

DOI: 10.1103/PhysRevE.95.032402

\section{INTRODUCTION}

In typical cell sorting experiments [1,2], a cell aggregate is formed with two different cell types, in different proportions. Usually endodermal cells are the minority type, while the majority is made of ectodermal cells. The experiments proceed as endodermal cells spontaneously migrate and segregate forming multiple clusters that diffuse and grow by merging with other clusters of their kind. The final configuration is a roughly round endodermal aggregate surrounded by ectodermal cells [1-4].

Usually the monitored quantities are the time to segregate and some characterization of the final pattern, i.e., some measure that tells whether the segregation is complete or not and/or whether the ectodermal cells completely involve the endodermal ones $[3,5]$.

There are different hypotheses regarding the possible mechanisms to explain this spontaneous segregation. It could be explained by assuming that cells differently attach to each other depending on their type, a theory known as differential adhesion hypothesis (DAH) [4], developed by Steinberg in 1963 and inspired by immiscible fluids behavior. Recently this hypothesis has been generalized to include cell cortex tension [6,7]. Another possibility considers that the cells capabilities of compressing and squeezing between its neighbors have different intensities depending on the cell type. This is the differential surface contraction hypothesis (DSCH), enunciated by Harris in 1976 [8] and further developed by Brodland [9]. By this hypothesis, cell sorting is driven

\footnotetext{
*Corresponding author: leon@if.ufrgs.br
}

by cell surfaces differential contraction, depending on the cell neighborhood. Yet another explanation was proposed by Jones et al. [3], by assuming that differences in the cell motility, together with cell recognition ability, would drive cell segregation. This last hypothesis is known as "different velocities hypothesis" (DVH). Typically, the experimental measures that support either of these models consider the final configuration, where the endodermal cells cluster is surrounded by ectodermal cells. These measures, however, are incapable to decide whether one or all of these mechanisms is behind cell segregation nor give information on time scales.

In dilute systems the mechanisms for cluster growth are clusters diffusion and cluster merging when they collide. If merging is faster than diffusion, we can assume that diffusion defines cluster growth time scales. Furthermore, in case cluster diffusion is normal with its diffusion constant scaling with the inverse of the mass, we expect that the average cluster mass grows as $t^{d /(d+2)}[10,11]$. Consequently, explanations for deviations from this theoretical prediction should be based on cluster diffusion properties.

More recent experiments on cell segregation monitored the evolution of both endodermal cell cluster size and diffusion through the ectodermal cells environment [12-14]. In particular, experiments by Méhes et al., using a two-point correlation method, measured cluster size during segregation and verified that collective migration accelerates segregation rates. Since the cell-cell interaction hypotheses described above (DAH, DVH, DSCH) affect merging mechanisms and not diffusion exponents, it is not surprising that none of those hypotheses could account for the fast time scales as observed in Ref. [14]. What suggests, together with simulations of Refs. [15-17], that cell sorting cannot be explained based 
on single-cell properties only, but rather depends on the interaction with neighboring cells, emerging as a collective phenomenon.

However, the specific mechanism by which collective behavior enhances cell segregation remains elusive. It turns out that simulations of interactive elements come in handy to discriminate the effects of different ingredients in cell sorting evolution. Cell sorting may be simulated by passive particles using the Glazier-Graner-Hogeweg (GGH) [18], by active matter models using the Vicsek model [19] or yet considering mixtures of both kinds of particles [20,21]. These models allow to consider several cell segregation mechanisms, such as DAH [6,15,18,22] and DVH [16,23]. Among these, active matter models provide a better control on the collective behavior properties, yielding similar power laws for cluster interface evolution when considering DAH or DVH $[15,16]$. This suggests that the mechanisms underlying both hypotheses have similar effects on cluster diffusion and direction persistence. On the other hand, this result should be further verified by comparing with a theoretical inference for a finite-size effect: experiments of cell sorting involve finite numbers of cells (up to order $10^{5}$ ) and cells have finite sizes. So, deviations from the behavior of a system of infinite number of infinitesimal cells should be expected to affect cluster growth. Consequently, theoretical approaches should explicitly include finite-size effects.

Here we report active matter simulation results and propose an equation for the growth of a mean endodermal cell cluster that diffuses through ectodermal cells. The general idea is to test whether the collective properties could be responsible for the observed endodermal cluster-size evolution by altering cluster diffusion properties. In order to justify the diffusive approach used for the evolution equation, we simulate the dynamics of single clusters and determine the limits within which their movement can be considered diffusive. Also, we obtain a general dependency of the cluster diffusion coefficient with its mass. We validate these results by simulating cell sorting using the differential adhesion and velocity hypotheses in active matter models, and show that, depending only on collective parameters values, both hypotheses present similar exponents for cluster-size evolution.

Based on our simulation results we introduce an analytic approach for the growth of endoderm cells cluster, which allows us to explicitly consider finite-size effects. This approach is valid in the dilute limit, that is when the time for a cluster of endoderm cells to diffuse between collisions is much longer than its rounding time. Finally, we present the analytic solutions for the equation and compare with the simulation results. The quality of the fits allows us to conclude that cell sorting can be approached as a cluster-cluster fusion problem [10]. Furthermore, after discounting finite-size effects, segregation experiments as described above are useful to evince the collective roots and properties of the system as, for example, the effective capability of the cells to follow their neighbors.

Section II explains the models used in the simulations and shows the results for single-cluster diffusion and segregation. Section III describes the mean-cluster approach and compares its solution with the simulations. In Sec. IV we discuss the results and conclude.

\section{SIMULATIONS}

In this section we initially present the cell-sorting simulations alternatively assuming two mechanisms: cell adhesion differences and cell velocity differences. In the final subsection we detail single-cluster diffusion and its dependence on the number of particles.

The simulations are performed using an active matter model running in GPU cards. Each cell is represented by a selfpropelled particle (SPP) with constant speed such that the evolution of the $i$ th particle position $\vec{x}_{i}$ is given by

$$
\vec{x}_{i}(t+\Delta t)=\vec{x}_{i}(t)+\vec{v}_{i}(t) \Delta t,
$$

where $\vec{v}_{i}$ is the particle's velocity [24]. The particle's direction at $t=t+\Delta t$ is

$$
\theta_{i}(t+\Delta t)=\arg \left\{\sum_{j \in \mathcal{V}_{i}}\left[\alpha^{\prime} \frac{\vec{v}_{j}(t)}{v_{0}}+\beta_{i j} \vec{f}_{i j}(t)\right]+\eta \vec{u}_{i}(t)\right\} .
$$

In Eq. (2), parameter $\alpha^{\prime}$ regulates the tendency of cells to follow the velocity direction of their neighbors; we call the normalized value $\alpha^{\prime} / v_{0}$ simply as $\alpha$. $\mathcal{V}_{i}$ comprises those cells that interact with cell $i ; \beta_{i j}$ is the intensity of the force between cells; $\vec{f}_{i j}$ expresses the dependence of the force on the distance between cells $i$ and $j$ shown in Eq. (3) below; $\vec{u}_{i}$ is a unitary vector that, together with the fixed intensity $\eta$, represents a directional noise acting on the system.

The force $\vec{f}_{i j}$ consists of a hard-core repulsion for distances between particles $i$ and $j$ smaller than a radius $r_{c}$ added to a harmonic-like interaction around the equilibrium radius $r_{e}$, ranging from $r_{c}$ up to a maximum $r_{0}$ :

$$
f_{i j}=\left\{\begin{array}{cl}
\infty & \text { if } r_{i j} \leqslant r_{c} \\
1-\frac{r_{i j}}{r_{e}} & \text { if } r_{c}<r_{i j}<r_{0} \\
0 & \text { if } r_{i j} \geqslant r_{0}
\end{array}\right.
$$

Consequently, all particles laying within a radius $r_{0}$ from particle $i$ comprise its neighborhood $\mathcal{V}_{i}$.

In what follows, we express all distances in units of $r_{e}$, with $r_{0}=1.32 r_{e}$, chosen to guarantee that each particle has six neighbors on average. The other parameters are $r_{c}=0.50 r_{e}$ and $v_{0}=0.0175 r_{e} / \Delta t$. Particles should not have very large speeds, in order to guarantee that the distance covered in one time step is much less than the core radius, $r_{c}$, ensuring that particles do not pass through each other. The largest speed used in simulations is $4 v_{0}[15,16,24]$. Also, the core forces are large and repulsive with values at least 1000 times larger than the remaining ones.

The simulations consider two types of particles, 1 (typically endodermal) and 2 (typically ectodermal), with speed and adhesion intensities for each particle depending on its type. We denote particle types by super-indexes and particle labels by subindexes. DAH [15] supposes differential adhesion so we keep the same speed for both cell types, that is, $v^{1}=v^{2}=v_{0}$, while we assumed type-dependent adhesion values: $\beta^{11}=$ 0.45 and $\beta^{22}=0.225$ to account for adhesion between same type cells while $\beta^{21}=\beta^{12}=0.24$ for intertype interactions, assumed to be symmetric. Similarly, DVH [16] assumes different velocities and hence we consider type-dependent 
(1)

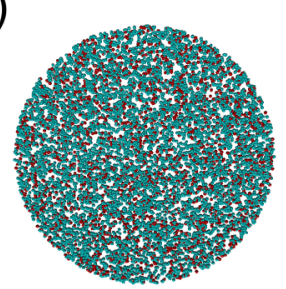

(2)

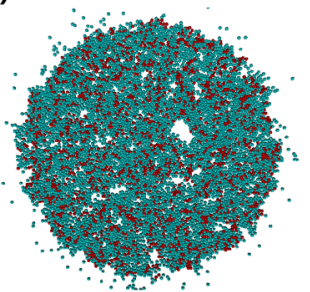

(3)

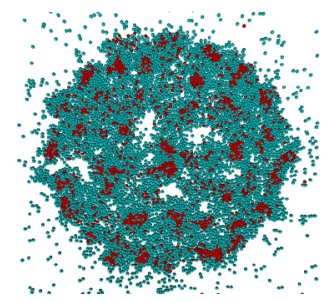

(4)

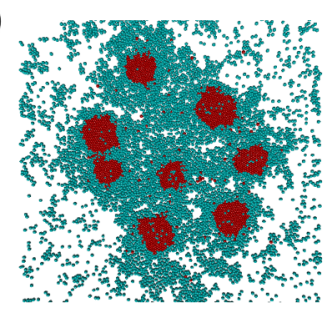

(5)

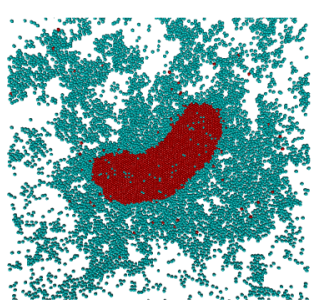

(6)

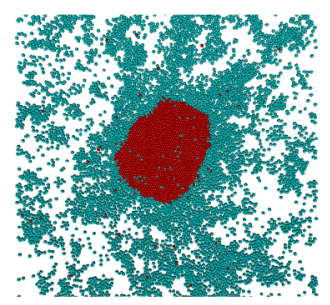

FIG. 1. Snapshots for a DVH simulation, the cyan (or lighter shade) cells are the faster ones. (1) Random initial state; (2) $t=10^{4} \Delta t$; (3) $t=10^{5} \Delta t$, clusters become visible; (4) $t=10^{7} \Delta t$; (5) $t=10^{8} \Delta t$, one large cluster, yet not spherical; (6) Final state with $t=3 \times 10^{8} \Delta t$, just one large circular cluster and some isolated endoderm cells.

speeds, that is, $v^{1}=v_{0}$ and $v^{2}=4 v_{0}$, while the adhesion is type-independent: $\beta^{11}=\beta^{12}=\beta^{21}=\beta^{22}=0.55$. All simulations are performed in a two-dimensional space, with up to $N=8000$ particles $(N=6400$ only in the $\alpha=0 \mathrm{DAH}$ case). The proportion of endoderm to ectoderm particles is $1: 3$, which avoids initial percolation of particles of type 1 , endodermal cells.

\section{A. Cell sorting simulations}

Cell-sorting simulations begin with cells of both types randomly placed in a circle of radius $\sim R_{c}$ located on a square domain of size $L$ and larger than the surface occupied by cells: $L^{2} \gg N \pi r_{e}^{2} \equiv \pi R_{c}^{2}$. Figures 1 and 2 show typical snapshots of the segregation evolution presenting successive increase in the sizes of type 1 particle clusters, for DVH and DAH, respectively.

We measured the average cluster size, $m$, using a cluster counting algorithm (detailed in the Appendix) and disregarding small clusters (less than 5 cells). Similar procedure is used by Méhes et al. [14] in their experiments. Figure 3 shows the mean-cluster evolution for typical samples of DVH and DAH for $\alpha=0$, that is, in the absence collective motion terms in Eq. (2). The DAH curve shown in Fig. 3 corresponds to a simulation with $N=6400$ particles while the DVH curve corresponds to $N=8000$. In both cases the proportion of

(1)

(3)
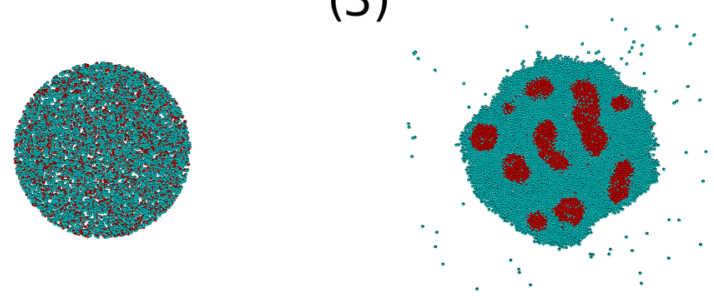

(2)

(4)

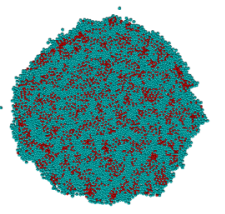

FIG. 2. Snapshots for a DAH simulation, the cyan (or lighter shade) cells are the less adhesive ones. (1) Random initial state; (2) $t=$ $10^{5} \Delta t$, clusters become visible; (3) $t=10^{7} \Delta t$; (4) $t=10^{8} \Delta t$, there is only a single cluster, yet not spherical, and some loose endoderm cells.

type 1 particles (endoderm) to type 2 particles (ectoderm) is $1: 3$. This means that the clusters of endoderm cells saturate around 1500 particles. The initial average cluster size is determined by the random configuration at $t=0$. The dashed lines are meant to evince the slope $\lambda=\frac{1}{2}$, which indicates the power-law trend after the initial transient and before the final saturation regimes. At long times the number of clusters is small and fusion events are rare, and that is what causes the discrete steps in $m(t)$ for $t \geqslant 10^{7}$.

\section{B. Diffusion of single clusters}

To evince the relation between collective motion and cluster diffusion, we simulate the diffusion of a single cluster of

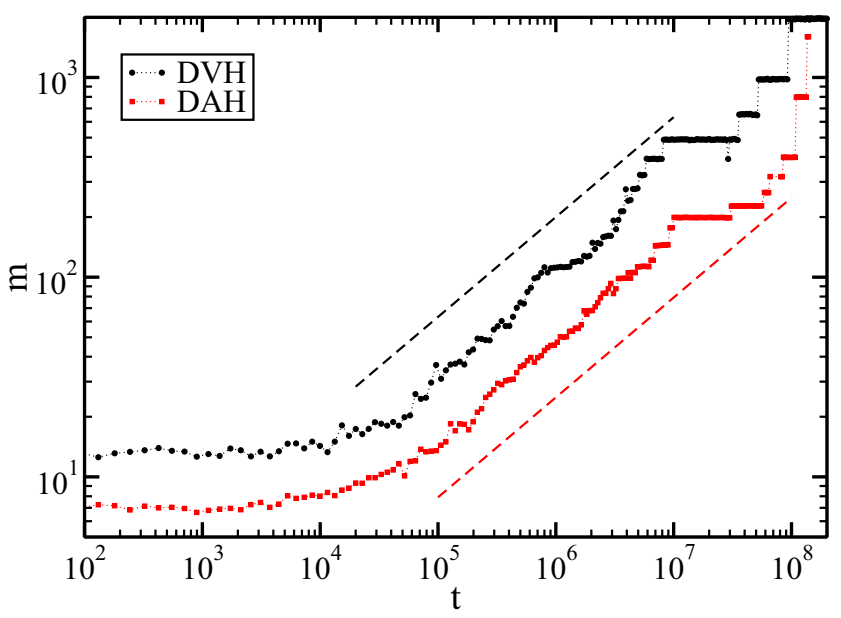

FIG. 3. Mean-cluster evolution for typical samples of DVH (black circle) and DAH (red square). Dashed lines, both with slope $\lambda=\frac{1}{2}$, indicate the tendency after an initial transitory and before final saturation. 


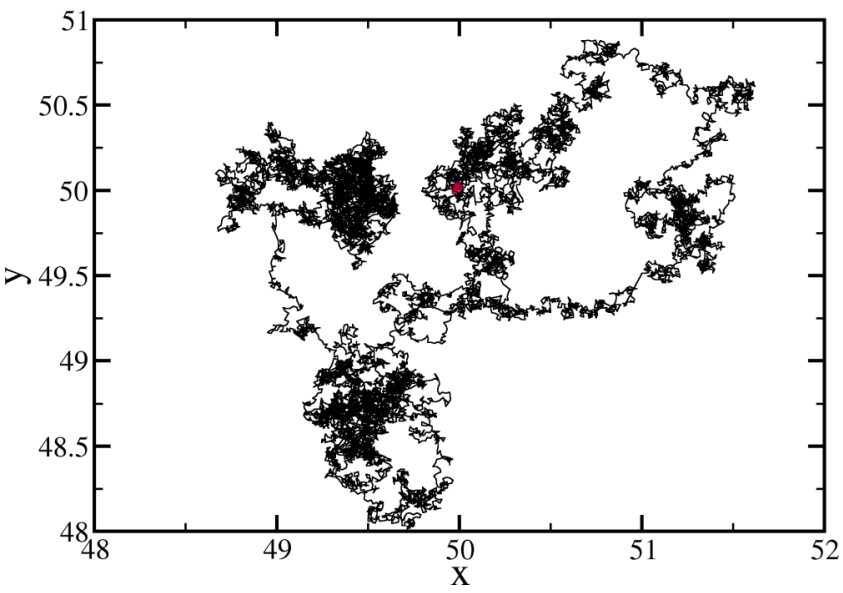

FIG. 4. Black lines show the trajectory of a single cluster of size 10 and $\alpha=0$ during $10^{7} \Delta t$.

particles and explore the relation between its mass and the alignment parameter $\alpha$. Figure 4 shows the trajectory of the center of mass of a single cluster with 10 particles and null alignment term $(\alpha=0)$.

The cluster diffusion is characterized by the mean-square displacement curve, obtained by first considering the position of the center of mass at time $t$ and its position at time $t+T$, where $T$ is a time window, ranging from zero to the duration of the simulation. Then the square displacement is calculated for every time $t$ and their average is calculated for each fixed time window $T$. The result is a set of values representing the mean-square displacement, $\left\langle\Delta r^{2}\right\rangle$, as a function of time window $T$.

Figure 5 shows plots of mean-square displacements $\left\langle\Delta r^{2}\right\rangle$ for $\alpha=0,5,10,15$ considering in each graph clusters of different sizes. In all cases we find normal diffusion after a small transitory. That is, the square displacement grows linearly with $T$. We obtain the diffusion coefficient, $D$, by finding the intercept of the asymptotic fit function at $T=1$
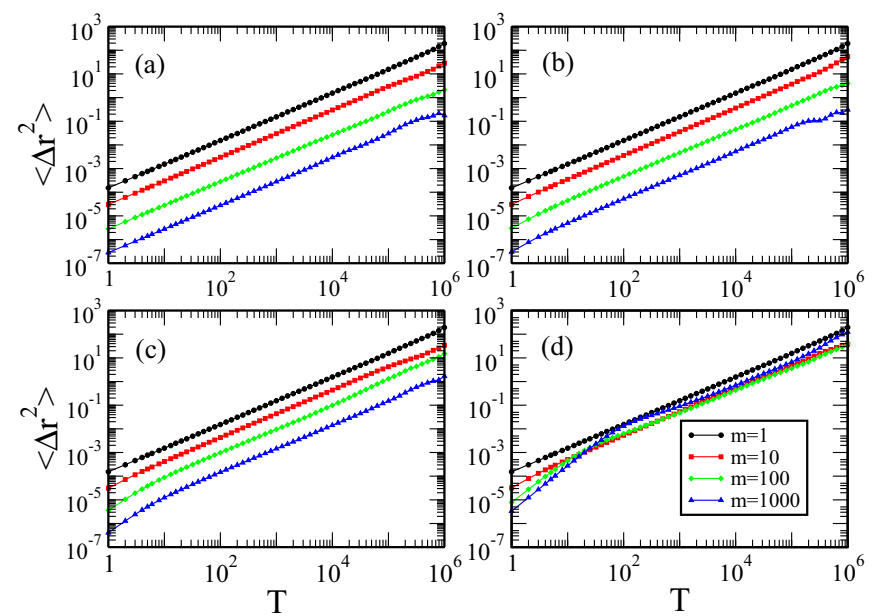

FIG. 5. Mean-square displacement $\left\langle\Delta r^{2}\right\rangle$ of single clusters of size $m=1,10,100,1000$ for different values of alignment parameter: (a) $\alpha=0$ (no alignment); (b) $\alpha=5$; (c) $\alpha=10$; (d) $\alpha=15$ (close to the transition to collective behavior).

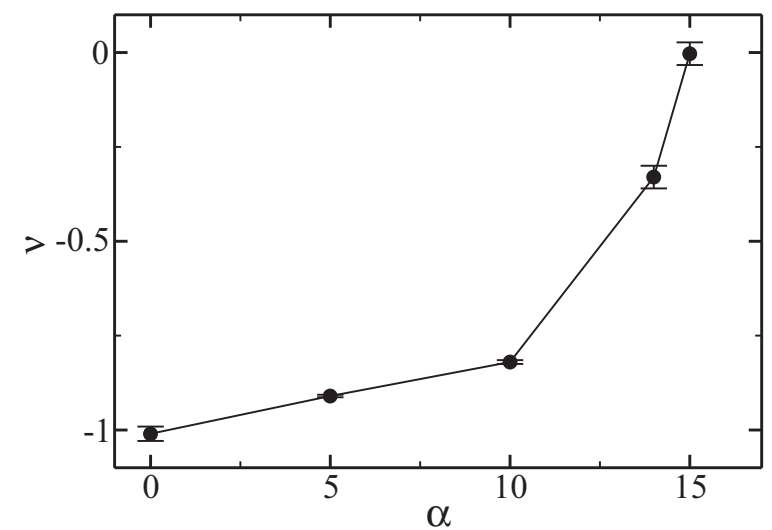

FIG. 6. Dependence of the diffusion mass exponent $v$ with the alignment term $\alpha$. With no alignment $(\alpha=0)$ we have the normal decay $v=-1$. Near the transition for the collective movement $(\alpha=$ 15) we find $v \sim 0$, meaning that clusters of all sizes move with the same diffusion constant.

for the different cluster mass values. For $\alpha=0$ [Fig. 5(a)] we find that $D$ is inversely proportional to the cluster mass, as expected,

$$
D(m) \propto m^{v}
$$

and $v \sim-1$ for $\alpha=0$.

As the alignment term increases [Figs. 5(b)-5(d)] deviations from normal diffusion are observed for small time windows $T$. However, the asymptotic behavior for large $T$ is normal in all cases studied in this work (Fig. 5). As previously, parameter $v$ can be determined using Eq. (4), but now with values different from -1 .

As shown in Fig. 6, the dependence of the diffusion coefficient with cluster mass changes as $\alpha$ increases. The exponent goes from $v \sim-1$ for $\alpha=0$ to $v \sim 0$ when $\alpha=15$. The deviations from the linear regime at short time windows can be better seen in Fig. 5(d): they are associated with a ballistic regime [25] with a corresponding characteristic persistence length of the cluster displacement. The largest persistence length found happens at $\alpha=15$ and for cluster sizes of 1000 particles. As can be seen in Fig. 5(d), this length is of the order of $0.1 r_{e}$, much less than the characteristic size $\left(r_{e}\right)$ of a single particle. This means we can neglect the ballistic regime in the analytic approach developed in the next section.

\section{MEAN-CLUSTER MODEL}

Snapshots of cell-sorting experiments [14] and simulations $[15,16]$ show that the growth of clustered structures happens through fusion of diffusing clusters with similar sizes, suggesting a scaling state as predicted by Kolb [10]. This regime holds far from the finite-size limits and at low concentration of endoderm cells. Considering only irreversible fusion the growth rate of an average cluster with $m$ cells can be described by

$$
\frac{d m}{d t}=\frac{m}{\tau(m)},
$$

where $\tau$ is a characteristic time for a cluster of endoderm cells (from now on, clusters, for simplicity) to diffuse and 
merge to another one. As showed in the previous section, we may consider normal diffusion for clusters even for $\alpha=15$, close to the transition to collective behavior [24]. So we may consider the characteristic time for cluster fusion as depending on the square of the average distance between clusters, $L$, and on the diffusion constant $D$, that is, $\tau \propto L^{2} / D$.

In systems with constant number of particles of each type, with global density $\rho$ and volume $V$, the typical distance between clusters of size $m$ is $L=(m / \rho)^{1 / d}$, where $d$ is the system dimension. Here, as in Ref. [10], we use $D$ proportional to some power of the average cluster mass, $D \propto m^{v}$ [Eq. (4)]. In the case of normal diffusion and particles with no alignment term, $v=-1$ [26]. However, as shown in the previous section, nontrivial $v$ values are found in systems with $\alpha \neq 0$ : although diffusion is normal.

Using this, we find $\tau=a m^{\frac{2-d v}{d}} \rho^{-\frac{2}{d}}$, with $a$ being a constant that includes the collision geometry terms, assumed to be time independent since (i) the number of cells is conserved and (ii) the cluster radius and the average distances between clusters are in direct proportion, so the angular fraction occupied by neighboring clusters on a spherical shell around a cluster is also conserved. Injecting this into Eq. (5) and solving for $m$ we obtain

$$
m(t)=\sqrt[2-d v]{\left(a^{\prime} t+m_{0}^{\frac{2-d v}{d}}\right)^{d}} .
$$

Here $a^{\prime} \equiv\left(\frac{2-d v}{d}\right) a$. For $a^{\prime} t \gg m_{0}$, we reobtain Kolb's results [10] for nonfractal clusters:

$$
m(t) \sim t^{d /(2-d v)} \equiv t^{\lambda} .
$$

Note that the power-law exponent depends only on the system dimension and on the exponent, $v$, relating diffusion and cluster mass. The solution in Eq. (6) does not consider upper or lower limits for cluster size, and we refer to it as the unbounded solution, since $m$ could vary from zero to infinity. However, we remark that it depends on the initial cluster mass $m_{0}$ that cannot be set to zero.

Now we focus on how the finite-size limitations change the evolution of the average cluster size. These are included by considering two limits for the average cluster size:

(i) a minimum cluster size, $c$, below which clusters do not grow;

(ii) a maximum cluster size, $b$, due to the finite number of cells.

This is included in Eq. (5) as follows:

$$
\frac{d m}{d t}=\frac{m-c}{\tau(m)}\left(1-\frac{m}{b}\right) .
$$

We refer to Eq. (8) as the mean-cluster approach (MCA). A general implicit solution for this is reported in the Appendix, the particular cases, $(v=-1, v=0)$, for which the solutions may be expressed as regular geometrical functions, are also shown in the Appendix.

The fixed point in Eq. (8) at $m=c$ is unstable since $d m / d t$ is negative for $m<c$ and positive for $m>c$. To avoid a trivial solution, the initial condition $m_{0}>c$ must hold. Solutions for the mean-cluster evolution are shown in Fig. 7, together with the solutions for the unbounded case given by Eq. (6). Note that (a) when $c=0$ there is a time lag to enter the scaling regime,

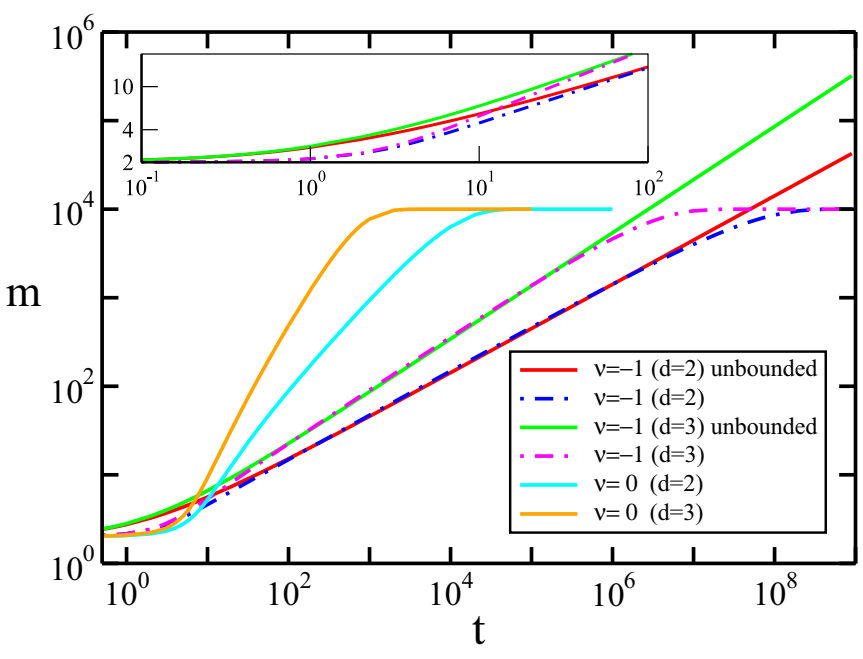

FIG. 7. Average cluster-size solution in d dimensions, for finite and unbounded systems, varying $v$. At long times unbounded systems converge to a power law. When in scaling regime the system with $v=-1$ has exponent $\lambda=1 / 2(\lambda=3 / 5)$ in $2 \mathrm{D}$ (3D) and with $v=0$ the exponents are $\lambda=1(\lambda=3 / 2)$. In all plots $a=1, b=$ $10^{4}, c=1.9$ and $m_{0}=2$. The inset details the effect of the minimum cluster for $v=-1$. Legends are the same as in the main graph.

related to the finite initial cluster size $m_{0}$; (b) when $c \lesssim m_{0}$ the time lag is longer but after it the slope is steeper than for the solution with $c=0$ (inset in Fig. 7); (c) depending on these limits, the region where the slopes of bounded and unbounded cases coincide may be limited to a few time decades. In this figure, it comprises around four decades when $v=-1$ and two decades when $v=0$; (d) for $v=-1$, the case with the same exponent of normal diffusion of particles without alignment term, the unbounded solution asymptotically converges to a power law with exponent $\lambda=\frac{1}{2}(\lambda=3 / 5)$ in $2 \mathrm{D}(3 \mathrm{D})$; (e) for $v$ $=0$ the exponents for the unbounded case are $\lambda=1(\lambda=3 / 2)$.

We compare the analytic model with simulations averaging over four samples for each set of parameters. In the simulations we use 2000 endoderm particles; this way we fix the correspondent parameter $b=2000$. The average initial cluster size, $m_{0}$, is directly measured for each sample using a cluster counting algorithm (see Appendix and Ref. [27]). The final time is around $t_{f} \sim 10^{8}$. However, at these long times there are too few clusters to obtain reliable averages and we stop the simulations with less than five clusters. While $b$ and $m_{0}$ are fixed by initial conditions, $a$ and $c$ are obtained by minimizing square deviations. The last parameter, $v$, was taken from the single clusters diffusion simulations previously discussed (see Fig. 6).

The violet triangles pointing left in Fig. 8 refer to DVH simulations data taken from Ref. [16], while other symbols refer to newly simulated data assuming DAH. The corresponding $\alpha$ values used in the simulations are also indicated in the legend box. As shown previously (see Fig. 6) each $\alpha$ value may be mapped to a single $v$ value. The MCA solution [Eq. (A2)] uses this $v$ value to fit the data with $a$ and $c$ left as free parameters. The fitting values are shown in Table I. These solutions are shown by the continuous lines in Fig. 8. To highlight the scaling properties seen in Eq. (6) we used reduced units $t^{*}=a t$ and $m^{*}=m / m_{0}$. Note the strong dependence of the average 


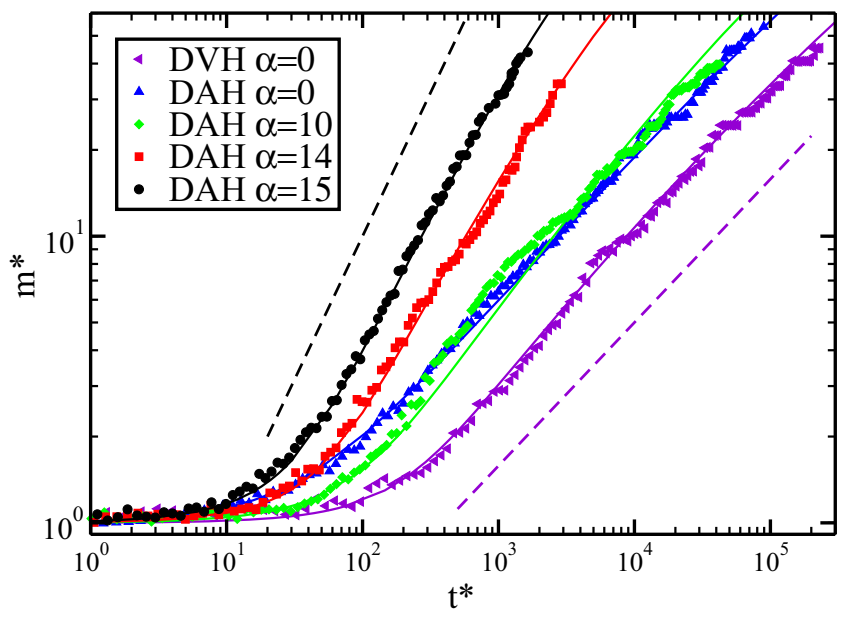

FIG. 8. Evolution of the average cluster size, both time and $m$ are given in reduced units, $t^{*}=a t$ and $m^{*}=m / m_{0}$. Each point results from an averaging over four runs. Lines indicate fits using MCA. Each $v$ value used in the fit is related to a specific value of $\alpha$ as shown in Fig. 6. The dashed lines are power laws to guide the eyes with exponent $\lambda=0.5$ (violet) and $\lambda=1.0$ (black).

cluster growth with $\alpha$ : segregation times may vary in more than two decades. The dashed lines in the figure represent two limits of Eq. (7): $\lambda=1 / 2$ (violet) and $\lambda=1$ (black), corresponding, respectively, to the diffusion exponents $v=-1$ and $v=0$. The quality of these fits indicate that MCA correctly explains the evolution of cell segregation for both DVH and DAH.

\section{DISCUSSION AND CONCLUSION}

Using simulations and a mean-cluster approach we showed that in two dimensions the exponent $\lambda$ associated to the growth of the average cluster mass, $m$, may exceed the expected classical value $1 / 2$ (or $3 / 5$ in three dimensions) [11,28,29], depending on the intensity of the collective dynamics. These results are in agreement with the experimental works by Méhes and collaborators, which reported values up $\lambda \sim 1.48$ for the cluster mass growth exponent in a mixture of fish keratocytes [14].

Previous simulation works $[15,16]$ measured a different parameter $(\gamma)$, related to the interface between tissues, and reported that it decreases in time with exponent $\sim-0.2$. In two dimensions these different measures are related by $m \propto \gamma^{-2}$, resulting in an equivalent value of $\lambda \sim 2 / 5$ for these simulations. The discrepancy can be explained if we consider that in the latter the small clusters are neglected, a

TABLE I. Fitted values for the free MCA parameters $a$ and $c$ for different values of the alignment parameter $\alpha$. Left column indicates the cell-sorting mechanism.

\begin{tabular}{lrcr}
\hline \hline & $\alpha$ & $a$ & $c$ \\
\hline DVH & 0.0 & $9.5 \times 10^{-3}$ & 11.5 \\
DAH & 0.0 & $1.0 \times 10^{-2}$ & 0.7 \\
DAH & 10.0 & $7.0 \times 10^{-4}$ & 3.3 \\
DAH & 14.0 & $2.8 \times 10^{-4}$ & 0.0 \\
DAH & 15.0 & $3.4 \times 10^{-4}$ & 9.5 \\
\hline \hline
\end{tabular}

procedure also adopted in the experiment reported in Ref. [14]. In the case with no alignment $(\alpha=0)$, simulations both with DAH and DVH find the same exponent $\lambda \sim 1 / 2$. When close to the the transition to collective behavior of the Vicsek model the exponent is $\lambda=1$ (Fig. 6), a region not explored in Refs. [15,16]. Mones and collaborators [17] obtained further results by simulating segregation in self-propelled particle systems. In agreement with our results, they showed that cluster growth exponents may largely deviate from the classical expected value of the Cahn-Hilliard equation [30] when velocity alignment is present.

The single cluster simulations showed that the ballistic regime, responsible for the migration persistence, exists only for short displacements. The largest ballistic displacements are shown by large clusters in systems close to the transition to collective behavior, extending for less than a particle diameter. In the diffusive regime that follows, we find a one to one correspondence between the alignment term, $\alpha$, and the exponent, $v$, relating cluster diffusion constant and cluster mass as $D \propto m^{\nu(\alpha)}$. This property explains the faster segregation rates: for large alignment tendency, as the clusters grow their diffusion coefficient is not reduced as much as expected for clusters formed by nonactive particles.

In the mean-cluster approach, this relation establishes a direct connection between the exponent $(\lambda)$ of the cluster-size evolution and the alignment parameter: in the scaling regime the alignment $\alpha$ is the single parameter determining cluster growth exponent. That is, MCA provides a theoretical prediction for the possible cluster growth exponents independently of the details of physical mechanisms (DVH, DAH, or others): the only necessary hypotheses for dilute systems are cluster irreversible fusion and diffusion.

The finite size of cells and minimal cluster sizes are responsible for the deviation from the power-law growth and their inclusion in the MCA evolution equation helps to find the correct rescaling of time and cluster mass. With this, simulation data in DAH and DVH become well described for time intervals varying from three to five decades, including both initial growth and power-law regimes.

The MCA fit of the simulation allows us to see the phenomena and the previous simulations [14,15,17] from another point of view, suggesting that the cell tendency to follow the neighborhood induces collective movement and greatly enhances cell segregation [17]. For $v=0$, MCA yields $\lambda=1$ in $2 \mathrm{D}$, which is in agreement with the linear trend experimentally found by Méhes et al. [14]. It also confirms the simulation results by Kabla [23], which stressed that the maximum segregation efficiency happens close to the streaming transition.

Experiments measuring either the relation of cluster diffusion constant and cluster mass, or simply cluster growth in systems with diluted endoderm cells, would be welcomed to confirm the basic scaling hypothesis underlying cluster growth and its dependence with collective motion.

\section{ACKNOWLEDGMENTS}

We thank the Brazilian agencies CAPES, CNPq, and FAPERGS for financial support. We also acknowledge the anonymous referees for their important contribution in the readability of this work. 


\section{APPENDIX}

\section{Solution of the mean-cluster equation}

A general implicit solution for Eq. (8) is written as $t=G(m)-G\left(m_{0}\right)$, where $G(x)$ is

$$
\begin{aligned}
G(x)= & \frac{b d x^{2 / d-v}}{a(c-b)(v d-2)}\left\{\left(\frac{b}{x-b}+1\right)_{2}^{v-2 / d} F_{1}[v-2 / d, v-2 / d ; v-2 / d+1 ;-b /(x-b)]\right. \\
& \left.-\left(\frac{x}{x-c}\right)_{2}^{v-2 / d} F_{1}[v-2 / d, v-2 / d ; v-2 / d+1 ;-c /(x-c)]\right\},
\end{aligned}
$$

and ${ }_{2} F_{1}$ is the Gauss hypergeometric series [31].

The solutions for the unbounded case are analytic and present explicit expressions as shown in the main article. Here we present the transcendental expressions derived from the hypergeometric equations solving Eq. (7), which includes both lower and upper bounds for two and three dimensions. For each dimension two values of exponent $v$ were considered, namely, $v=$ $-1,0$. The first value represents the case of normal diffusion and the other is the case where the diffusion is independent of the cluster mass.

Solutions in two dimensions for $v=-1$ :

$$
G(m)=\frac{b}{a(b-c)}\left\{-\frac{1}{2}\left[2 b m+m^{2}+2 b^{2} \ln (|b-m|)\right]+\frac{1}{2}\left[m^{2}+2 c^{2} \ln (|m-c|)\right]\right\} .
$$

Solution in two dimensions for $v=0$ :

$$
G(m)=\frac{1}{a(b-c)}\left[-b^{2} \ln (|b-m|)+b c \ln (|m-c|)\right] .
$$

Solution in three dimensions for $v=-1$ :

$$
\begin{aligned}
G(m)= & \frac{b}{2 a(b-c)}\left\{b^{5 / 3} \ln \left(b^{2 / 3}+b^{1 / 3} m^{1 / 3}+m^{2 / 3}\right)-2 b^{5 / 3} \ln \left(b^{1 / 3}-m^{1 / 3}\right)-2 \sqrt{3} b^{5 / 3}\left[\operatorname{arctg}\left(\frac{\frac{2 m^{1 / 3}}{b^{1 / 3}}+1}{\sqrt{3}}\right)\right]-3 b\left(m^{2 / 3}\right)\right. \\
& \left.-c^{5 / 3} \ln \left(c^{2 / 3}+c^{1 / 3} m^{1 / 3}+m^{2 / 3}\right)+2 c^{5 / 3} \ln \left(c^{1 / 3}-m^{1 / 3}\right)+2 \sqrt{3} c^{5 / 3}\left[\operatorname{arctg}\left(\frac{\frac{2 m^{1 / 3}}{c^{1 / 3}}+1}{\sqrt{3}}\right)\right]+3 \mathrm{~cm}^{2 / 3}\right\} .
\end{aligned}
$$

Three-dimensional solution for $v=0$ :

$$
\begin{aligned}
G(m)= & \frac{1}{2 a(b-c)}\left[\left(b^{1 / 3} \ln \left(b^{2 / 3}+\sqrt[3]{b m}+m^{2 / 3}\right)+\frac{2 b \ln \left(b^{1 / 3}-m^{1 / 3}\right)}{c^{2 / 3}}+2 b^{1 / 3} \ln \left(b^{1 / 3}-m^{1 / 3}\right)\right.\right. \\
& \left.+2 \sqrt{3} b^{1 / 3} \tan ^{-1}\left(\frac{2 \sqrt[3]{m}+\sqrt[3]{b}}{\sqrt{3} \sqrt[3]{b}}\right)-\frac{b \ln \left(c^{2 / 3}+\sqrt[3]{c m}+m^{2 / 3}\right)+2 b \ln \left(c^{1 / 3}-m^{1 / 3}\right)}{c^{2 / 3}}+2 \sqrt{3} b^{1 / 3} \tan ^{-1}\left(\frac{2 \sqrt[3]{m}+\sqrt[3]{c}}{\sqrt{3} \sqrt[3]{c}}\right)\right] .
\end{aligned}
$$

In the limits of $b \rightarrow \infty$ and $c \rightarrow 0$ all these solutions converge to Eq. (6) of the main paper. The correct results are attained in two dimensions under expansion of the logarithmic functions up to second order. In three dimensions the arc tangent and the logarithm must be expanded to fifth order.

The hypergeometric function is defined as

$$
{ }_{2} F_{1}\left[k_{1}, k_{2} ; l_{1} ; z\right]=\sum_{i=0}^{\infty} \frac{\left(k_{1}\right)_{i}\left(k_{2}\right)_{i} z^{k}}{\left(l_{1}\right)_{i}},
$$

with the condition

$$
|z|<1
$$

and also

$$
(q)_{n}=\left\{\begin{array}{cc}
1 & n=0 \\
q(q+1) \ldots(q+n-1) & n>0 .
\end{array}\right.
$$

\section{Cluster-counting algorithm}

We define a cluster as the set of particles of a given kind where each one has at least one neighbor of this same kind at maximal distance $r_{0}$. We start labeling all particles from 1 to $N$ and defining an adjacency matrix (AM) [32]. This is an $N \times N$ matrix $A$ whose generic element $A_{i j}$ is 1 if the distance between particles with labels $i$ and $j$ are smaller than $r_{0}$, else $A_{i j}$ is zero. The algorithm proceeds as follows [27]:

(1) Loop over line 1 of AM until $A_{1 j}=1$ is found at column $j$.

(2) All elements of line $j$ are added to line 1.

(3) First element of line $j$ is set to -1 .

(4) Repeat the first and second steps until the last column.

(5) Find the next line with a non-negative first element, say line $i$.

(6) Repeat steps 1 to 5 for line $i$.

(7) Repeat until the end of lines. 
The number of clusters can be found in the resulting matrix counting the number lines starting with nonnegative values.
The number of particles on each cluster is found by simply adding the values of these nonnegative lines.
[1] J. Holtfreter, Rev. Can. Bio. 3, 220 (1944).

[2] H. V. Wilson, J. Exp. Zool. 5, 245 (1907).

[3] B. M. Jones, P. M. Evans, and D. A. Lee, Exp. Cel. Res. 180, 287 (1989).

[4] M. S. Steinberg, Science 141, 401 (1963).

[5] R. A. Foty and M. S. Steinberg, Dev. Biol. 278, 255 (2005).

[6] M. L. Manning, R. A. Foty, M. S. Steinberg, and E. M. Schoetz, Proc. Natl. Acad. Sci. USA 107, 12517 (2010).

[7] J. D. Amack and M. L. Manning, Science 338, 212 (2012).

[8] A. K. Harris, J. Theor. Biol. 61, 267 (1976).

[9] G. W. Brodland, J. Biomech. Eng. 124, 188 (2002).

[10] M. Kolb, Phys. Rev. Lett. 53, 1653 (1984).

[11] A. Nakajima and S. Ishirara, New J. Phys. 13, 033035 (2011).

[12] M. Krieg, Y. Arboleda-Estudillo, P. H. Puech, J. Käfer, F. Graner, D. J. Müller, and C. P. Heisenberg, Nat. Cell Biol. 10, 429 (2008).

[13] A. V. Klopper, G. Krens, S. W. Grill, and C.-P. Heisenberg, Eur. Phys. J. E 33, 99 (2010).

[14] E. Méhes, E. Mones, V. Nemeth, and T. Vicsek, PLoS ONE 7, e31711 (2012).

[15] J. M. Belmonte, G. L. Thomas, L. G. Brunnet, R. M. C. de Almeida, and H. Chaté, Phys. Rev. Lett. 100, 248702 (2008).

[16] C. P. Beatrici and L. G. Brunnet, Phys. Rev. E 84, 031927 (2011).

[17] E. Mones, A. Czirók, and T. Vicsek, New J. Phys. 17, 063013 (2015).
[18] F. Graner and J. A. Glazier, Phys. Rev. Lett. 69, 2013 (1992).

[19] T. Vicsek, A. Czirok, E. Ben-Jacob, I. Cohen, and O. Shochet, Phys. Rev. Lett. 75, 1226 (1995).

[20] S. R. McCandlish, A. Baskaran, and M. F. Hagan, Soft Matter 8, 2527 (2012)

[21] J. Stenhammar, R. Wittkowski, D. Marenduzzo, and M. E. Cates, Phys. Rev. Lett. 114, 018301 (2015).

[22] Y. Zhang, G. L. Thomas, M. Swat, A. Shirinifard, and J. A. Glazier, Plos One 6, e24999 (2011).

[23] A. J. Kabla, J. R. Soc. Interface 9, 3268 (2012).

[24] G. Grégoire, H. Chaté, and Y. Tu, Physica D 181, 157 (2003).

[25] G. Grégoire, H. Chaté, and Y. Tu, Phys. Rev. E 64, 011902 (2001).

[26] R. Kubo, M. Toda, and N. Hashitsume, Statistical Physics II (Springer-Verlag, Berlin, 1978), p. 6.

[27] M. A. Wong and T. Lane, J. Roy. Stat. Soc. Ser. B (Methodological) 45, 362 (1983).

[28] A. J. Bray, Adv. Phys. 51, 481 (2002).

[29] A. Sicilia, Y. Sarrazin, J. J. Arenzon, A. J. Bray, and L. F. Cugliandolo, Phys. Rev. E 80, 031121 (2009).

[30] A. J. Bray, Adv. Phys. 43, 357 (1994).

[31] M. Abramowitz and I. A. Stegun (eds.), Handbook of Mathematical Functions with Formulas, Graphs and Mathematical Tables (National Bureau of Standards, Washington, 1964), Chap. 15, p. 555.

[32] G. Chartrand, Introductory Graph Theory (Dover, New York, 1985), p. 218. 